\title{
Diagnostic accuracy and additional value of diffusion-weighted imaging for discrimination of malignant cervical lymph nodes in head and neck squamous cell carcinoma
}

\author{
R. B. J. de Bondt • M. C. Hoeberigs $\cdot$ P. J. Nelemans • \\ W. M. L. L. G. Deserno • C. Peutz-Kootstra • \\ B. Kremer • R. G. H. Beets-Tan
}

Received: 5 October 2008 / Accepted: 10 December 2008 / Published online: 10 January 2009

(C) The Author(s) 2009. This article is published with open access at Springerlink.com

\begin{abstract}
Introduction The aim was to determine the diagnostic accuracy and additional value of diffusion-weighted imaging for detection of malignant lymph nodes in head and neck squamous cell carcinoma.
\end{abstract}

R. B. J. de Bondt $(\bowtie) \cdot$ M. C. Hoeberigs $\cdot$ R. G. H. Beets-Tan Department of Radiology, Maastricht University Medical Center, P. Debyelaan 25,

6202 AZ Maastricht, The Netherlands

e-mail: rbj.de.bondt@mumc.nl

M. C. Hoeberigs

e-mail: mc.hoeberigs@mumc.nl

R. G. H. Beets-Tan

e-mail: r.beets.tan@mumc.nl

P. J. Nelemans

Department of Epidemiology,

Maastricht University Medical Center,

Maastricht, The Netherlands

e-mail: Patty.nelemans@epid.unimaas.nl

W. M. L. L. G. Deserno

Department of Radiology, Twenteborg Hospital,

Almelo, The Netherlands

e-mail: W.Deserno@zgt.nl

C. Peutz-Kootstra

Department of Pathology, Maastricht University Medical Center,

Maastricht, The Netherlands

e-mail: Carine.peutz@mumc.nl

B. Kremer

Department of Otorhinolaryngology/Head and Neck Surgery,

Maastricht University Medical Center,

Maastricht, The Netherlands

e-mail: Bernd.kremer@mumc.nl
Methods Two hundred nineteen lymph nodes, predominantly smaller than $10 \mathrm{~mm}(95.4 \%)$, in 16 consecutive patients were evaluated at $1.5 \mathrm{~T}$. Lymph nodes were evaluated for maximum short axial diameter, morphological criteria, and apparent diffusion coefficient (ADC) values ( $b=0$ and $b=1,000 \mathrm{~s} / \mathrm{mm}^{2}$ ). Sensitivity, specificity, positive and negative predictive values as well as diagnostic odds ratios (DORs) and areas under the curves (AUCs) of ROC curves were calculated for the various magnetic resonance imaging (MRI) criteria individually and in combination. Histological examination of lymph nodes in the neck dissection specimen was the gold standard to determine malignant involvement.

Results The optimal ADC threshold was $1.0 \times 10^{-3} \mathrm{~mm}^{2} / \mathrm{s}$. Using this cutoff point, sensitivity and specificity were $92.3 \%$ and $83.9 \%$, respectively. When used in combination with size and morphological criteria, ADC value $<1.0 \times$ $10^{-3} \mathrm{~mm}^{2} / \mathrm{s}$ was the strongest predictor of presence of metastasis (DOR=97.6). A model which added ADC values to the other MRI criteria performed significantly better than a model without $\mathrm{ADC}$ values: $\mathrm{AUC}=0.98$ versus $\mathrm{AUC}=0.91 \quad(p=0.036)$.

Conclusion In this study, with predominantly small lymph nodes, the ADC criterion is the strongest independent predictor of presence of metastasis. The use of ADC values in combination with the other MRI criteria significantly improves the discrimination between malignant and benign lymph nodes.

Keywords Head and neck · Lymph nodes · Metastases · MRI · Diffusion-weighted imaging 


\section{Introduction}

The presence of cervical lymph node metastases is an important prognostic factor in patients with head and neck squamous cell carcinoma (HNSCC) as this significantly worsens the treatment outcome [1-8]. Radiological imaging plays an increasingly important role in accurate assessment of the lymph node status of the neck.

Frequently used criteria for differentiation between benign and malignant lymph nodes are the presence of necrosis - mainly seen in larger lymph nodes - and assessment of lymph node morphology such as borders and internal heterogeneity $[9,10]$. Also the introduction of lymph node specific contrast agents like ultrasmall particles iron oxide on magnetic resonance imaging (MRI) provides differentiation between benign and malignant lymph nodes in the head and neck and results are improving [11-16].

Another commonly used criterion is the maximum short axial diameter for which several studies have been undertaken to determine the optimal cutoff size [17, 18]. Small lymph nodes with a maximum short axial diameter below $10 \mathrm{~mm}$ are more challenging for radiologists, because the mere use of this size criterion will result in misclassification of malignant lymph nodes as normal on MRI evaluation. According to the literature, the performance of MRI is still poor in the assessment of lymph node metastases, and this concerns especially the detection of metastases in small lymph nodes [11].

Diffusion-weighted imaging (DWI) is a noninvasive MRI technique that provides image contrast dependent on the molecular motion of water. Any architectural changes in the proportion of extracellular to intracellular water protons-like metastases in lymph nodes - will alter the diffusion coefficient of the tissue. Therefore, DWI provides in characterization of different tissues and lesions. Previous reports showed the utility of DWI in the region of the head and neck for different pathology [19-26]. In studies focused on nodal staging, the imaging results were based on series with only clinical positive necks in which large lymph nodes were selected for evaluation [27-30]. Studies focusing on mainly negative necks at clinical examination are scarce. The aim of this study was to prospectively determine the diagnostic accuracy and additional value of DWI-MRI to conventional MRI - using size and morphology criteria - for differentiation between malignant and normal cervical lymph nodes.

\section{Materials and methods}

\section{Patients}

The local medical ethics committee approved this prospective study. Between March 2006 and December 2007, a series of 16 consecutive patients with a HNSCC who were scheduled for a supraomohyoid neck dissection (SOHND), selective or radical neck dissection (RND) enrolled in this study. All patients underwent MR imaging as part of the routine diagnostic workup; the findings of diffusionweighted imaging were not used for clinical decision making. MR imaging was performed previous to ultrasonography with fine needle aspiration cytology of lymph nodes in the neck.

\section{MR imaging}

All examinations were performed at $1.5 \mathrm{~T}$ (Gyroscan, Powertrack 6000, Philips, Best, The Netherlands) using a Head-Neck coil (Philips, Best, The Netherlands). The Synergy Head-Neck coil was used for both the conventional and diffusion-weighted MR imaging; the range was from the base of skull to the level of the clavicles. To ascertain correlation of the conventional images and DWI, all sequences were acquired with similar geometry.

The conventional MR protocol consisted of axial fatsuppressed T2-weighted (TR/TE 1704/80), axial and coronal T1-weighted (TR/TE 400/12), contrast-enhanced axial and coronal fat-suppressed T1-weighted (TR/TE 740/ $12)$, and coronal short tau inversion recovery (TR/TE/TI $5868 / 60 / 15)$ sequences. Slice thickness was $3 \mathrm{~mm}$; there was no interslice gap. The matrix was $272 \times 512$ with a field of view 220 (rectangular field of view (RFOV) 80\%).

Diffusion-weighted images were acquired in the axial plane by using a gradient single-shot echo planar imaging sequence. The sequence was repeated for two $b$ values $b=0$ and $b=1,000 \mathrm{~s} / \mathrm{mm}^{2}$ with the following parameters: TR/TE $5666 / 70$, section thickness was $3 \mathrm{~mm}$ according to the conventional protocol, the matrix was $272 \times 512$ with a field of view 400 (RFOV 80\%), there was no interslice gap, and the number of signal acquisition was 6 . A total of 64 slices were acquired which covered the whole neck area from the skull base to the clavicles. The total scan time was 4 min 54 s. DWI was performed before the contrast-enhanced T1weighted sequences. Apparent diffusion coefficient (ADC) maps were automatically constructed by the postprocessing software of the MR system.

Image evaluation

Two radiologists with specific experience in head and neck imaging (12 and 4 years, respectively) and taking part of multidisciplinary head and neck oncology conferences in a referral hospital independently evaluated the conventional images. Both observers were blinded to each other's MR assessments and clinical information.

On the conventional MR images, lymph nodes were assessed for size and morphological criteria. All lymph 
nodes were determined by position and slice number per neck level, the maximum short axial diameter was recorded per lymph node, and lymph nodes were classified as normal $(\leq 10 \mathrm{~mm})$ or malignant $(>10 \mathrm{~mm})$. Furthermore, for each lymph node, it was predicted whether it was benign or malignant by using the following morphological criteria determined on the fat-suppressed T2-weighted images: the border of the lymph node (smooth, lobulated, spiculated, or indistinct). Presence of smooth and lobulated borders were considered as indicative of normal lymph nodes whereas that of spiculated and indistinct borders of malignant lymph nodes (Fig. 1). Another criterion was the degree of homogeneity of the signal intensity; homogeneous signal intensity was considered as normal whereas heterogeneous signal intensity was considered as indicative of malignant lymph nodes.

Diffusion-weighted images were evaluated on the MRI scanner workstation on which the ADC maps were constructed automatically by the postprocessing software of the MR system. In consensus, the two radiologists, who also assessed the conventional MR images, performed the evaluation of the diffusion-weighted images. To ascertain a node-by-node correlation between the conventional images versus the diffusion-weighted images, all lymph nodes on the T1-weighted and T2-weighted images were correlated to the corresponding b1000 images. On these b1000 images, a region of interest (ROI) was drawn manually around each single lymph node, the ROI was copied to the ADC map, and the ADC value was automatically calculated by the postprocessing software of the MR machine. This was done on all subsequent sections that contained lymph node parenchyma to ensure that most of the lymph node was included. The final ADC value of a lymph node was an average of all values from one single lymph node; this was expressed as mean \pm standard deviation. Figure 2 demonstrates an example of this postprocessing technique. A necrotic area in a lymph node, determined as a hyperintense region on the T2-weighted image with a hypointense region on the (contrast-enhanced) T1-weighted images, was excluded from the area of analysis.

\section{Pathological examination}

All neck dissections were performed "en bloc". Immediately after the resection, the surgeon positioned the neck

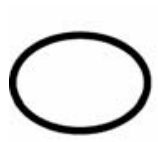

smooth

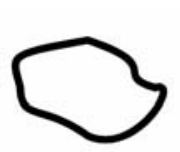

lobulated

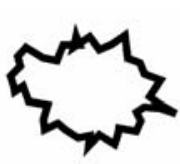

spiculated

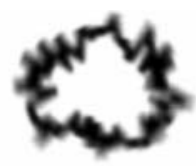

indistinct
Fig. 1 Schematic drawing of the four borders for assessment of cervical lymph nodes dissection specimen on a schematic drawing of the neck in real proportions and fixed it with needles. The pathologist manually identified and localized the lymph nodes per neck level in the specimen. The maximum short axial diameter of all lymph nodes was recorded. Subsequently, lymph nodes were fixed, sectioned, and hematoxylin-eosin (HE) stained and the presence of tumor in each lymph node was examined microscopically. The results at pathology were used as the reference standard.

\section{Pathological-radiological correlation}

The neck was subdivided according to the six different levels in agreement with the classification of the American Joint Committee on Cancer; this classification was used by the radiologists when viewing the MR images and used by the pathologist when interpreting the specimen after neck dissection [31].

The results of the measurements on MRI were compared with the results of the pathological examination of the neck dissection specimens. By recording the combination of the maximum short axial diameter and the exact location of each lymph node per neck level—related to surrounding anatomic structures such as blood vessels, muscles, and salivary glands - it was possible to perform a topographic correlation for each lymph node between the pathological examination and the MR images.

\section{Statistical analysis}

Interobserver agreement with respect to the evaluation of the criteria size (maximum short axial diameter), border irregularity, and homogeneity of signal intensity on T2-weighted images was expressed by Cohen's kappa-coefficient $(\kappa)$. For each lymph node, the scores on MRI criteria were correlated with the results of pathologic examination of the neck dissection specimen. Pathologic results with respect to presence or absence of lymph node metastasis were used as standard of reference. Results on MRI criteria were dichotomized and diagnostic accuracy was evaluated in terms of sensitivity, specificity, positive predictive value (PPV), and negative predictive value (NPV).

Furthermore, discriminative ability was expressed by the diagnostic odds ratio (DOR). The higher the DOR, the higher the ability of a particular criterion to discriminate between lymph nodes with and without metastasis. A DOR= 1 indicates that the criterion under study has no diagnostic value; a $\mathrm{DOR}>1$ indicates that the criterion has ability to discriminate between lymph nodes with and without metastasis.

The independent contribution of each criterion under study was analyzed using multivariate logistic regression models. In these models, presence or absence of metastasis 
Fig. 2 Overview of measurement of the apparent diffusion coefficient value of cervical lymph nodes. Axial T1-weighted (a) and fat-suppressed T2weighted (b) images are used to ascertain matching between lymph nodes (arrows) on the conventional and the diffusionweighted imaging. Two small lymph nodes (arrows) can also be observed on the DWI b1000 image (c); on this image regions of interest are drawn around the lymph nodes and automatically copied to the ADC map (d) on which reference numbers are correlated to the $\mathrm{ADC}$ values
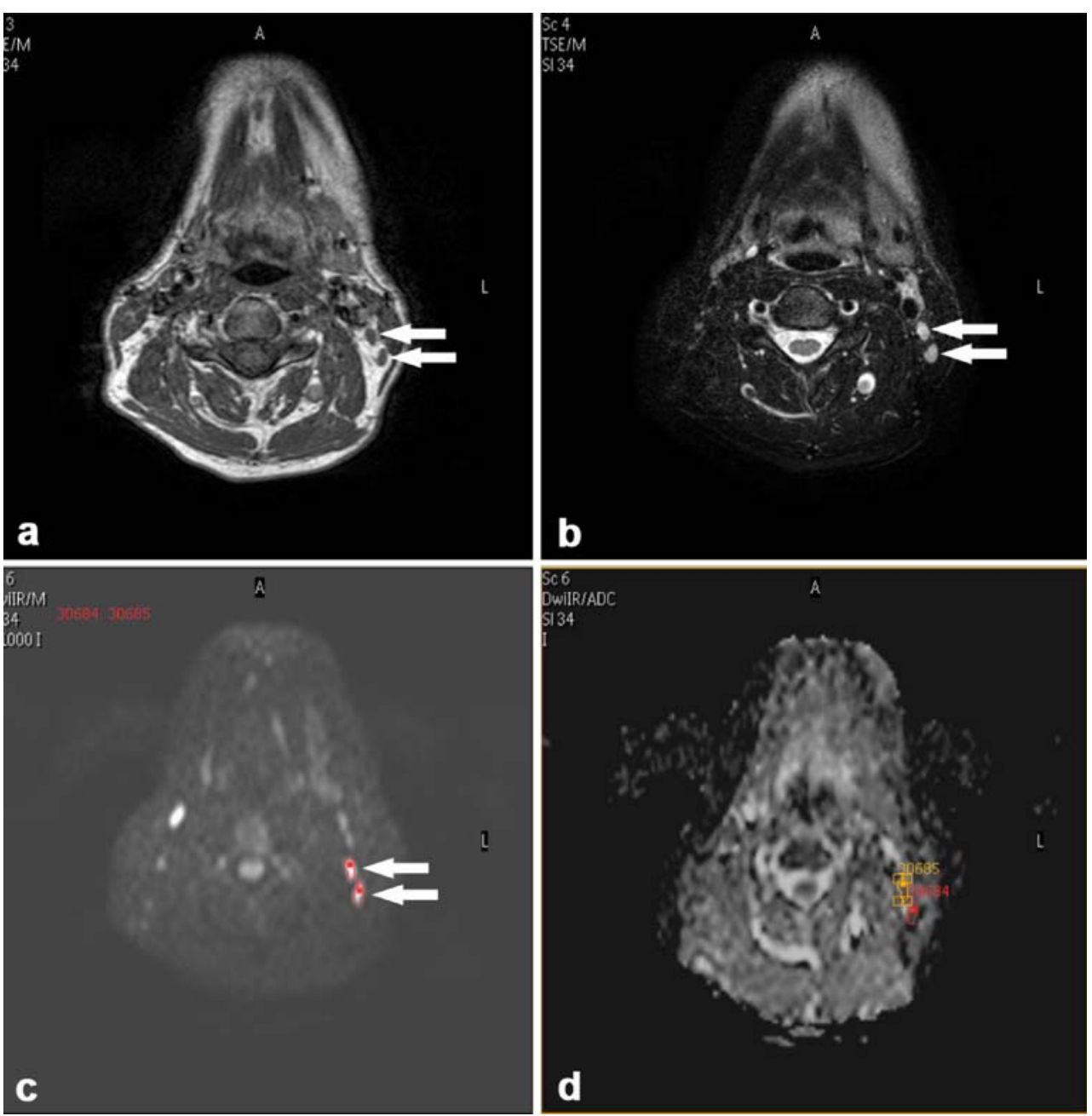

according to pathologic examination was the dependent variable and the dichotomized results on MRI criteria were entered as independent variables. Diagnostic odds ratios, which can be derived from these models by exponentiation of the regression coefficients, indicate to what extent each criterion in the model can be used as an independent predictor of the presence of metastasis in a lymph node.

Finally, the added diagnostic value of ADC values to the other MRI criteria (size, border irregularity, and signal intensity on T2-weighted images) was analyzed by using multivariate logistic regression models, which included different sets of MRI criteria. Model-derived predicted probabilities were used to construct receiver operating characteristic (ROC) curves. Areas under the curve (AUCs) with $95 \%$ confidence intervals were used to compare the diagnostic accuracy of various models. Differences between the AUCs were tested for statistical significance using the method described by Hanley and McNeil, which accounts for the fact that the AUCs are derived from the same sample of patients. Statistical analyses were performed with SPSS 13 and STATA. In all analyses, $p$ values $<0.05$ were considered as indicator for statistical significance [32].

\section{Results}

In 16 patients (mean age 59 years [range $40-77$ years]; seven women and nine men), a total of 17 neck dissections were performed: SOHND, $n=4$; RND, $n=4$; and selective neck dissection, $n=9$. Distribution of the total of 58 dissected neck levels was as follows: level I, $n=17$; level II, $n=17$; level III, $n=12$; level IV, $n=5$; and level V, $n=7$. Relevant patient characteristics, clinical findings, tumor-node-metastasis (TNM) classification and details of the performed neck dissections are summarized in Table 1.

At assessment of the MR images of the 58 dissected neck levels, a total of 281 lymph nodes were detected. Of these lymph nodes, 62 had a short axial diameter of $\leq 2 \mathrm{~mm}$. Because these lymph nodes were not detected at the pathological examination, they were excluded from further analysis. The large majority (13 out of 16) of patients presented without palpable lymph nodes at clinical examination (clinical negative neck); only a few lymph nodes exceeded the maximum short axial diameter of $10 \mathrm{~mm}$ at pathological and radiological examination. 
Table 1 Clinical findings in 16 consecutive patients with primary head and neck squamous cell carcinoma, clinical TNM classification, and details of the performed neck dissections.

\begin{tabular}{|c|c|c|c|c|c|c|c|c|}
\hline \multirow[t]{2}{*}{ Patient no. } & \multirow[t]{2}{*}{ Age (years) } & \multirow[t]{2}{*}{ Gender } & \multicolumn{2}{|l|}{ Clinical presentation } & \multirow[t]{2}{*}{ Clinical TNM } & \multicolumn{2}{|l|}{ Neck dissection } & \multirow[t]{2}{*}{ Dissected levels } \\
\hline & & & Primary tumor location & Side & & Type & Side & \\
\hline 1 & 64 & M & Tongue & $\mathrm{R}$ & T2N0M0 & Selective ND & $\mathrm{R}$ & I-III, V \\
\hline 2 & 75 & M & Nasopharynx & $\mathrm{L}$ & T2N1M0 & Radical ND & $\mathrm{L}$ & $\mathrm{I}-\mathrm{V}$ \\
\hline 3 & 53 & M & Tongue & $\mathrm{R}$ & T3N1M0 & Radical ND & $\mathrm{R}$ & $\mathrm{I}-\mathrm{V}$ \\
\hline 4 & 62 & $\mathrm{~F}$ & Tongue & $\mathrm{L}$ & T3N0M0 & Selective ND & $\mathrm{L}$ & I, II \\
\hline 5 & 58 & M & Vallecula & $\mathrm{R}$ & T1N0M0 & Selective ND & $\mathrm{R}$ & I, II, III, V \\
\hline 6 & 40 & M & Tongue & $\mathrm{L}$ & T2N0M0 & Selective ND & $\mathrm{L}$ & I-III \\
\hline 7 & 54 & M & Nasopharynx & $\mathrm{L}$ & T1N1M0 & Selective ND & $\mathrm{L}$ & I-III \\
\hline 8 & 63 & M & Retro molar trigonum & $\mathrm{L}$ & T2N0M0 & Selective ND & $\mathrm{L}$ & I-IV \\
\hline 9 & 55 & $\mathrm{~F}$ & Submandibular gland & $\mathrm{L}$ & T2N0M0 & Selective ND & $\mathrm{L}$ & I, II \\
\hline 10 & 72 & M & Tongue & $\mathrm{L}$ & T2N0M0 & Supraomohyoid ND & $\mathrm{L}$ & I-III \\
\hline 11 & 74 & $\mathrm{~F}$ & Cheek & $\mathrm{L}$ & T2N0M0 & Selective ND & $\mathrm{L}$ & I, II \\
\hline 12 & 51 & $\mathrm{~F}$ & Floor of mouth & $\mathrm{R}$ & T2N0M0 & Radical ND & $\mathrm{R}$ & $\mathrm{I}-\mathrm{V}$ \\
\hline 13 & 52 & $\mathrm{~F}$ & Soft palate & $\mathrm{L}$ & T2N0M0 & Radical ND & $\mathrm{L}$ & $\mathrm{I}-\mathrm{V}$ \\
\hline 14 & 47 & $\mathrm{~F}$ & Tongue & $\mathrm{R}$ & T3N0M0 & Supraomohyoid ND & $\mathrm{R}$ & I-III \\
\hline 15 & 50 & $\mathrm{~F}$ & Tongue & $\mathrm{R}$ & T2N0M0 & Selective ND & $\mathrm{R}$ & I, II \\
\hline \multirow[t]{2}{*}{16} & 77 & M & Nose & - & T2-3N0M0 & Supraomohyoid ND & $\mathrm{R}$ & I-III \\
\hline & & & & & & Supraomohyoid ND & $\mathrm{L}$ & I-III \\
\hline
\end{tabular}

$N D$ neck dissection, $L$ left, $R$ right, $M$ male, $F$ female

Pathological examination revealed 219 lymph nodes, which were matched to the lymph nodes on the MR images. Most lymph nodes were situated at level I $(n=45)$, level II $(n=100)$, or level III $(n=49)$. Only 25 nodes were detected at level IV $(n=7)$ or level V $(n=18)$. Evidence of metastases was demonstrated in 26 lymph nodes (node-based prevalence $=11.8 \%$ ) and in 11 of 16 patients (patient-based prevalence $=68.8 \%$ ). Lymph node size in relation to the presence of metastases $(n=26)$ was as follows: $5 \mathrm{~mm}, n=5$; $6 \mathrm{~mm}, n=4 ; 7 \mathrm{~mm}, n=3 ; 8 \mathrm{~mm}, n=6 ; 9 \mathrm{~mm}, n=1 ; 10 \mathrm{~mm}$, $n=3 ; 15 \mathrm{~mm}, n=1 ; 18 \mathrm{~mm}, n=1 ; 20 \mathrm{~mm}, n=1$; and $30 \mathrm{~mm}$, $n=1$. The majority $(95.4 \%)$ of lymph nodes on MRI showed a maximum short axial diameter $\leq 10 \mathrm{~mm}$. Only ten nodes $(4.6 \%)$ were $>10 \mathrm{~mm}$. Of these ten pathological determined lymph nodes, six were negative at pathological examination (range 11-12 $\mathrm{mm}$ in maximum short axial diameter). Four lymph nodes, with maximum short axial diameter ranging from $15-30 \mathrm{~mm}$, showed massive metastasis. Twenty-two of the 26 metastases were found in the lymph nodes $\leq 10 \mathrm{~mm}$. Figure 3 illustrates a lymph node with maximum short axial diameter of $12 \mathrm{~mm}$ with an ADC value of $1.3 \times 10^{-3} \mathrm{~mm}^{2} / \mathrm{s}$; pathological examination revealed no metastasis. Observer agreement for the three morphological criteria was $\kappa=0.86$ for size, $\kappa=1.00$ for border irregularity, and $\kappa=0.91$ for signal intensity on the T2-weighted images.

Table 2 presents combinations of sensitivity and specificity that were found using different cutoff values for ADC. Lower ADC values correspond with higher probability of lymph node metastasis. ADC values of malignant lymph nodes were significantly lower compared with benign lymph nodes with mean (standard deviation, SD) values of $0.85 \times 10^{-3} \mathrm{~mm}^{2} / \mathrm{s}\left(\mathrm{SD} 0.19 \times 10^{-3} \mathrm{~mm}^{2} / \mathrm{s}\right)$, and $1.2 \times 10^{-3} \mathrm{~mm}^{2} / \mathrm{s}\left(\mathrm{SD} 0.24 \times 10^{-3} \mathrm{~mm}^{2} / \mathrm{s}\right)$, respectively. High sensitivity combined with acceptable specificity was found for a cutoff point of $<1.0 \times 10$ versus $>1.0 \times 10^{-3} \mathrm{~mm}^{2} / \mathrm{s}$. This cutoff value was used for further analyses.

Table 3 shows the sensitivity, specificity, PPV and NPV for dichotomized ADC values, and the other MRI criteria such as size, border irregularity, and signal intensity on T2weighted images. Both the dichotomized size and ADC criteria are associated with the highest sensitivity, but the high sensitivity for size is compromised by a high proportion of false positive results.

Table 4 shows diagnostic odds ratios with $95 \%$ confidence intervals (CI) for the individual MRI criteria when they are used separately (derived from univariate analyses) and for the individual MRI criteria when they are used in combination with each other (derived from multivariate analyses). When used separately, the border criterion is associated with the highest ability to discriminate between malignant and benign lymph nodes $(\mathrm{DOR}=90.1)$. However, when used in combination, the ADC criterion is the strongest independent predictor of presence of metastasis ( $\mathrm{DOR}=97.6)$ followed by the border criterion $(\mathrm{DOR}=56.0)$. When evaluated in combination with the other criteria, the DOR of 4.4 (with $95 \%$ CI 0.7-28.0) associated with size no longer differs significantly from 1 and thus has no independent predictive value.

Table 5 presents areas under the curve with $95 \%$ confidence intervals from various models. Model 3, adding ADC values to the other MRI criteria (size, border irregularity, and 
Fig. 3 Fifty-one-year-old patient with a squamous cell carcinoma in the floor of the mouth on the left side (arrowheads). The axial T1-weighted (a) and fat-suppressed T2-weighted (b) images show in level II on the left side an enlarged lymph node (arrow) with a maximum short axial diameter of $12 \mathrm{~mm}$. This lymph nodes is easily recognized on the diffusion-weighted image at b1000 (c). The corresponding area on the apparent diffusion coefficient map (d) shows a calculated ADC of $1.3 \times 10^{-3} \mathrm{~mm}^{2} / \mathrm{s}$. Pathological examination revealed no metastasis
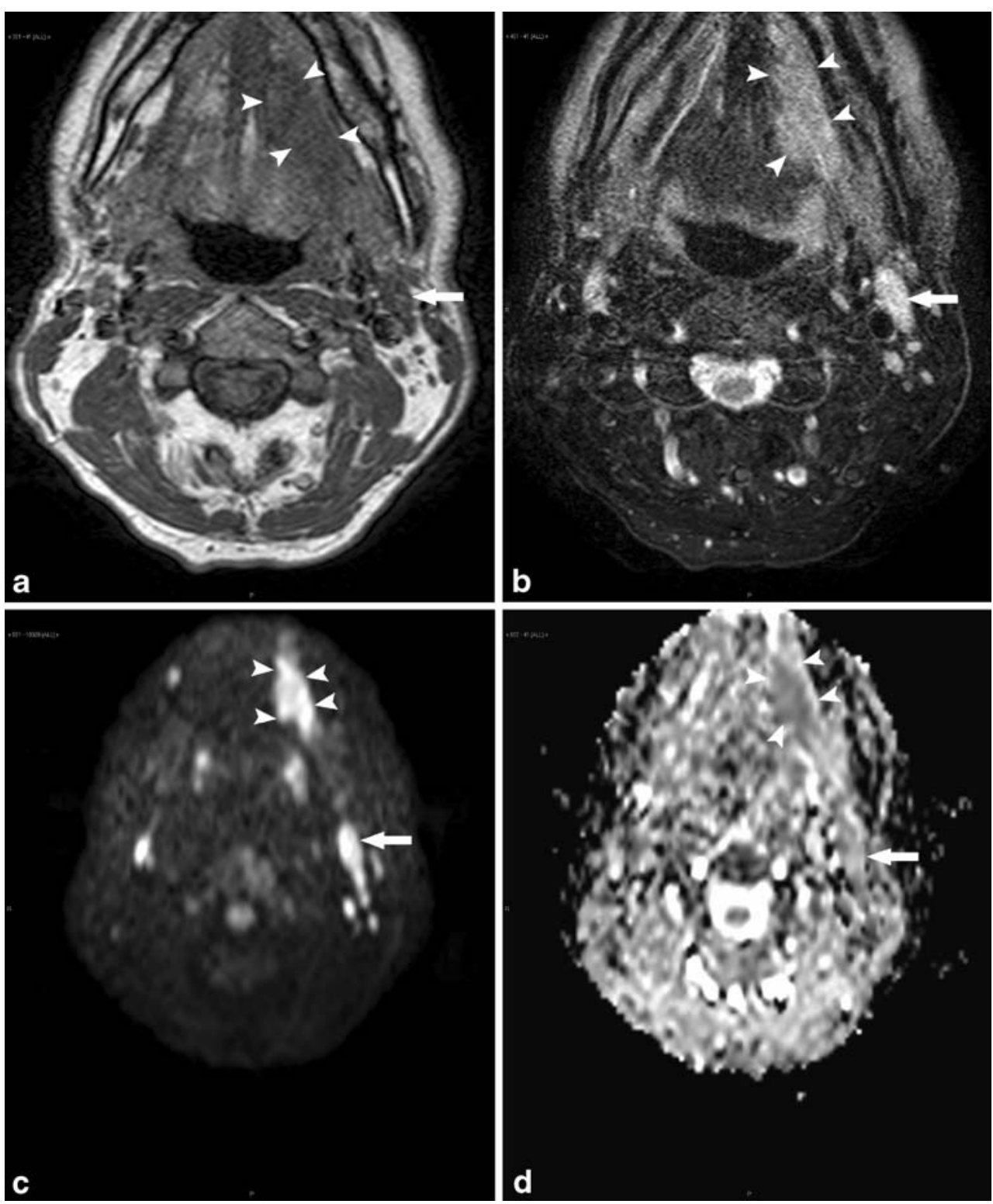

signal intensity on T2-weighted images), performs significantly better than model 2 that does not add ADC values to the other three criteria: $\mathrm{AUC}=0.98$ versus $\mathrm{AUC}=0.91(p=0.036)$. Moreover, model 1, which includes only ADC as predictor of presence of metastasis, performs nearly as well as model 2, which includes the other three MRI criteria as predictors: $\mathrm{AUC}=0.89$ versus $\mathrm{AUC}=0.91(p=0.52)$.

\section{Discussion}

The results show that apparent diffusion coefficient values calculated on the diffusion-weighted images, alone or in combination with other morphological criteria, are useful in discriminating malignant from benign lymph nodes in head and neck squamous cell carcinoma. ADC values of

Table 2 Sensitivity, specificity, and diagnostic odds ratio with $95 \%$ confidence interval for the diagnosis of malignant cervical lymph nodes for different threshold values of the apparent diffusion coefficient.

\begin{tabular}{lccc}
\hline Threshold & Sensitivity (\%) & Specificity (\%) & DOR (95\% CI) \\
\hline$<0.9 \times 10$ versus $>0.9 \times 10^{-3} \mathrm{~mm}^{2} / \mathrm{s}$ & 69.2 & 92.2 & $26.7(10.0-71.5)$ \\
$<0.94 \times 10$ versus $>0.94 \times 10^{-3} \mathrm{~mm}^{2} / \mathrm{s}$ & 84.6 & 90.2 & $50.4(15.7-161.6)$ \\
$<1.0 \times 10$ versus $>1.0 \times 10^{-3} \mathrm{~mm}^{2} / \mathrm{s}$ & 92.3 & 83.9 & $62.7(14.1-279)$ \\
\hline
\end{tabular}


Table 3 Sensitivity, specificity, positive predictive value, and negative predictive value for the diagnosis of malignant lymph nodes for the MR criteria: apparent diffusion coefficient $(\leq 1.0 \times 10$ versus $>1.0 \times 10^{-3} \mathrm{~mm}^{2} / \mathrm{s}$ ), size (maximum short axial diameter $\leq 10$ versus $>10 \mathrm{~mm}$ ), border irregularity and signal intensity on T2weighted images.

\begin{tabular}{|c|c|c|c|c|}
\hline Criterion & Sensitivity $(\%)$ & Specificity $(\%)$ & PPV $(\%)$ & NPV $(\%)$ \\
\hline $\operatorname{ADC}\left(<1.0 \times 10\right.$ versus $\left.>1.0 \times 10^{-3} \mathrm{~mm}^{2} / \mathrm{s}\right)$ & 92.3 & 83.9 & 43.6 & 98.8 \\
\hline Size $(\leq 10$ versus $>10 \mathrm{~mm})$ & 92.3 & 60.6 & 24.0 & 98.3 \\
\hline $\begin{array}{l}\text { Border irregularity on } \mathrm{T} 2 \text {-weighted images (spiculated/indistinct } \\
\text { versus smooth/lobulated) }\end{array}$ & 61.5 & 98.3 & 84.2 & 94.4 \\
\hline SI on T2-weighted images (heterogeneous versus homogeneous) & 50.0 & 94.8 & 56.5 & 93.3 \\
\hline
\end{tabular}

$P P V$ positive predictive value, $N P V$ negative predictive value, $A D C$ apparent diffusion coefficient, $S I$ signal intensity

malignant lymph nodes were significantly lower compared to benign lymph nodes.

At present, there are only few reports that demonstrate the usefulness of DWI-MRI in characterizing lymph nodes in the neck. Some studies reported the ability of DWI to discriminate between different histological lymph node cancers in the neck [28-30]. Others showed that ADC determination on DWI-MRI may have added value in discriminating malignant from benign lymph nodes in the neck $[19,20,27]$. All studies were performed in patients with palpable cervical lymphadenopathy (clinical positive necks), and further evaluation by MRI was limited to selected enlarged lymph nodes.

In contrast to previous reports, this study is unique in that it mainly dealt with patients with clinically negative necks. In only three out of 16 patients lymph nodes were palpable at clinical examination; however, in only two patients an enlarged lymph node with maximum short axial diameter ranging from 18 to $30 \mathrm{~mm}$ could also be demonstrated on MRI and at pathological examination. The large majority of lymph nodes $(95.4 \%)$ on MRI were $\leq 10 \mathrm{~mm}$; within this group, the pathologically confirmed malignant lymph nodes ranged a maximum short axial diameter of 5-10 $\mathrm{mm}$. Those small lymph nodes would all be assigned as benign based on the size criterion. Especially the multivariate regression analyses demonstrate that the size criterion no longer differs significantly malignant from benign lymph nodes and thus has no independent predictive value. When used separately, the border criterion is associated with the highest ability to discriminate between lymph nodes with and without metastasis; however, when used in combination, the ADC criterion is the strongest independent predictor of presence of metastasis followed by the border criterion (Table 4).

These results in the clinically negative necks are of great clinical relevance as DWI shows to be an accurate tool to differentiate metastases in small lymph nodes. In a radiologically negative neck, the planned neck dissection could be converted to a wait-and-scan policy, whereas the radiologically determined positive neck would support the decision to perform a neck dissection. The relative high sensitivity and NPV for the size criterion in this study only accounts for lymph nodes exceeding $10 \mathrm{~mm}$ in maximum short axial diameter (Table 3). However, as in this study, the large majority of lymph nodes were $\leq 10 \mathrm{~mm}$; the size criterion does no longer differ in these lymph nodes between malignant and benign lymph nodes. This is expressed by the low DOR for the size criterion in the univariate and multivariate logistic regression analyses (Table 4).

The optimal ADC threshold for this study population appeared to be $1.0 \times 10^{-3} \mathrm{~mm}^{2} / \mathrm{s}$ (Fig. 4); when the criteria size and morphology on the conventional MR protocol were combined with this ADC threshold value, the area

Table 4 Diagnostic odds ratios with $95 \%$ confidence intervals for apparent diffusion coefficient levels and three assessed morphological criteria (size, border, and signal intensity).

\begin{tabular}{llr}
\hline MRI criteria & $\begin{array}{l}\text { Univariate analysis } \\
\text { DOR (95\% CI) }\end{array}$ & $\begin{array}{c}\text { Multivariate analysis } \\
\text { DOR (95\% CI) }\end{array}$ \\
\hline ADC threshold $\left(<1.0 \times 10\right.$ versus $\left.>1.0 \times 10^{-3} \mathrm{~mm}^{2} / \mathrm{s}\right)$ & $62.7(10.0-71.5)$ & $97.6(9.7-982)$ \\
Size $(\leq 10$ versus $>10 \mathrm{~mm})$ & $18.5(4.2-80.4)$ & $4.4(0.7-28.0)$ \\
Border irregularity $($ spiculated/indistinct versus smooth/lobulated) & $90.1(22.5-361)$ & $56.0(4.4-719)$ \\
Signal intensity on T2-weighted image (heterogeneous versus homogeneous) & $18.2(6.7-49.4)$ & $12.8(1.8-91.6)$ \\
\hline
\end{tabular}

Results are derived from univariate and multivariate logistic regression analyses

$D O R$ Diagnostic odds ratio, $A D C$ apparent diffusion coefficient 
Table 5 Area under the curve and 95\% confidence intervals for predicted probabilities of malignant lymph nodes derived from four models which included different combinations of MRI criteria.

\begin{tabular}{llrr}
\hline Model & Criteria & AUC & 95\% CI \\
\hline 1 & ADC (alone) & 0.89 & $0.86-0.92$ \\
2 & Size, border, and signal intensity on T2-weighted images & 0.91 & $0.84-0.99$ \\
3 & ADC, size, border, and signal intensity on T2-weighted images & 0.98 & $0.97-1.00$ \\
4 & Size and ADC & 0.94 & $0.91-0.96$ \\
\hline
\end{tabular}

$A U C$ area under the curve, $A D C$ apparent diffusion coefficient

under the curve significantly increased. Figure 5 illustrates the ROC curves derived from predicted probabilities from models including different criteria sets. A model which added ADC values to the other MRI criteria performed significantly better than a model without ADC values whereas the model which includes only ADC performs nearly as well as the model which includes the other three MRI criteria. In a previous study, the same assessed morphological criteria showed-in addition to the size criterion - an increased AUC from 0.91 to 0.96. However, this accounted solely for the experienced head and neck radiologist in a series with higher prevalence of metastases $(27.2 \%)$ and larger assessed lymph nodes $(18 \%>10 \mathrm{~mm})$ [33].

The chosen ADC threshold of $1.0 \times 10^{-3} \mathrm{~mm}^{2} / \mathrm{s}$ in this study might be difficult to reproduce in other centers. This is probably based on the differences in study design and techniques that will be used and differences in selection of patient series, i.e., the sizes of assessed lymph nodes might influence the choice of the ADC threshold as larger lymph nodes tend to show necrosis and this will affect the ADC

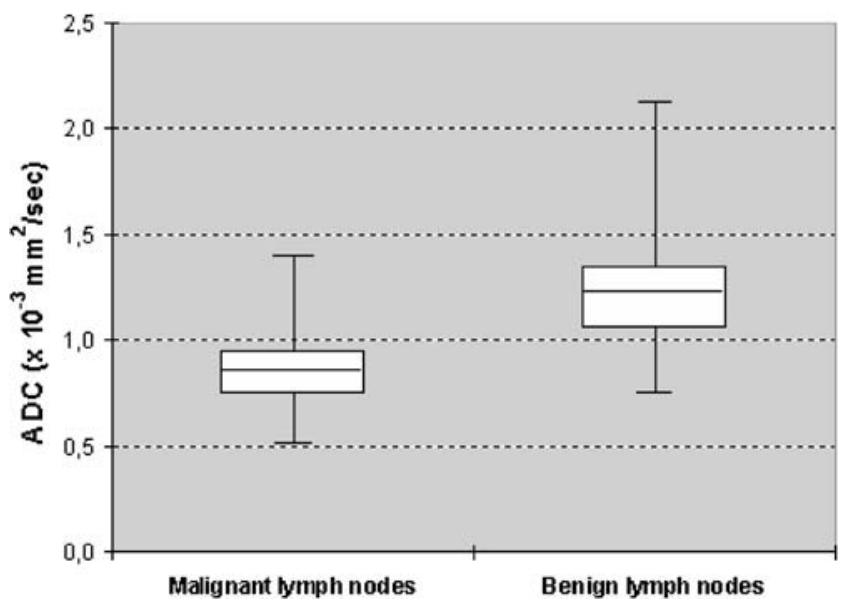

Fig. 4 Graph of box plots shows the apparent diffusion coefficient $(A D C)$ values of malignant and benign lymph nodes. The horizontal line in the box represents the median (50th percentile), whereas the top and the bottom represent the 25 th and 75 th percentile, respectively. The whiskers represent the range from the largest and the smallest measured ADC data. The ADC values of benign lymph nodes are higher than compared than those of malignant lymph nodes values. To use DWI for characterizing lymph nodes in general practice, there should be a well-established threshold for the ADC values. It is advisable that thresholds are reproducible in different centers and on different MR machines, to set the ADC threshold to the proper value depends on the differential diagnosis of expected lymph node pathology.

Some limitations of this study have to be addressed. Firstly, the low prevalence of malignant lymph nodes, with as a result that the negative predictive values were high for all MRI criteria. It must be kept in mind that predictive values are not independent of the setting in which the test is used and are strongly influenced by the prevalence; metastases were present in only 26 out of 219 lymph nodes

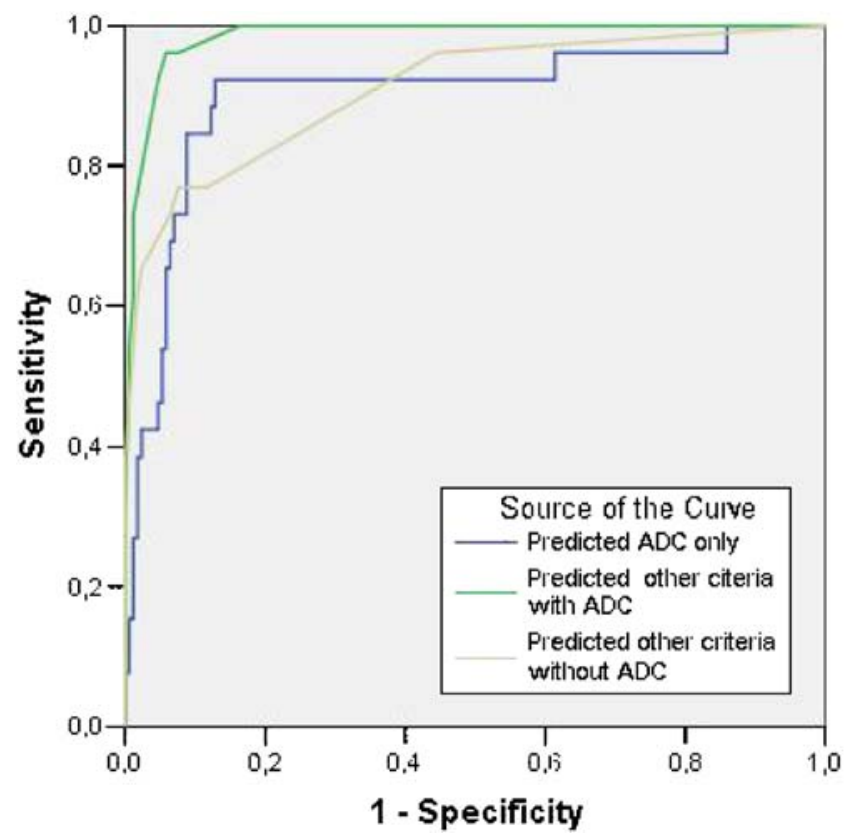

Fig. 5 Receiver operating characteristics curves derived from predicted probabilities from models including different sets of MRI. A model which added ADC values to the other MRI criteria (green line) performed significantly better than a model without $\mathrm{ADC}$ values (gray line $): \mathrm{AUC}=0.98$ versus $\mathrm{AUC}=0.91(p=0.036)$. Moreover, the model which includes only $\mathrm{ADC}$ (blue line) as predictor of presence of metastasis performs nearly as well as the model, which includes the other three MRI criteria as predictors: $\mathrm{AUC}=0.89$ versus $\mathrm{AUC}=0.91$ $(p=0.52)$ 
and negative predictive values of tests tend to be high when applied in a population with a low likelihood of the disease. It also has to be mentioned that the primary tumor sites in this study, which are relatively more likely to show small metastases, have been of influence especially on the size criterion showing low diagnostic accuracy and additional value. Secondly, in nine out of 45 lymph nodes (20\%) with a size of 3-4 $\mathrm{mm}$ in maximum short axial diameter, ADC values were observed below the threshold of $1.0 \times 10^{-3} \mathrm{~mm}^{2} / \mathrm{s}$. None of these small lymph nodes showed histopathological evidence of metastasis; partial volume effect when drawing ROIs on the b1000 images around these small lymph nodes might be responsible for the false positive results. Thirdly, although the scan time of DWI will only take less than $5 \mathrm{~min}$, it must be said that evaluating all lymph nodes at the MR workstation is time consuming.

\section{Conclusions}

We demonstrated in a series with predominantly small lymph nodes that ADC values calculated on the diffusion-weighted MRI is a strong predictor of the presence of metastasis, independently from other predictor such as size and morphology, in patients with head and neck squamous cell carcinoma (HNSCC). The use of ADC values in combination with the other MRI criteria significantly improves the discrimination between malignant and benign lymph nodes.

Conflict of interest statement We declare that we have no conflict of interest.

Open Access This article is distributed under the terms of the Creative Commons Attribution Noncommercial License which permits any noncommercial use, distribution, and reproduction in any medium, provided the original author(s) and source are credited.

\section{References}

1. Kalnins IK, Leonard AG, Sako K, Razack MS, Shedd DP (1977) Correlation between prognosis and degree of lymph node involvement in carcinoma of the oral cavity. Am J Surg 134:450-454

2. Jakobsen J, Hansen O, Jorgensen KE, Bastholt L (1998) Lymph node metastases from laryngeal and pharyngeal carcinomascalculation of burden of metastasis and its impact on prognosis. Acta Oncol 37:489-493

3. Kehrl W, Wenzel S, Niendorf A (1998) Effect of various forms of metastatic lymph node involvement on prognosis of squamous epithelial carcinomas of the upper aerodigestive tract. Laryngorhinootologie 77:569-575

4. Ganzer U, Meyer-Breiting E, Ebbers J, Vosteen KH (1982) Effect of tumor size on lymph node metastasis and type of treatment on the prognosis of hypopharyngeal cancer. Laryngol Rhinol Otol (Stuttg) 61:622-628
5. Leemans CR, Tiwari R, Nauta JJ, van der Waal I, Snow GB (1994) Recurrence at the primary site in head and neck cancer and the significance of neck lymph node metastases as a prognostic factor. Cancer 73:187-190

6. Leemans CR, Tiwari RM, van der Waal I, Karim AB, Nauta JJ, Snow GB (1992) Neck lymph node dissection in squamous cell carcinoma originating in the head-and-neck area; the significance for the prognosis. Ned Tijdschr Geneeskd 136:221-225

7. Foote RL, Olsen KD, Davis DL (1993) Base of tongue carcinoma: patterns of failure and predictors of recurrence after surgery alone. Head Neck 15:300-307

8. Taylor JM, Mendenhall WM, Lavey RS (1991) Time-dose factors in positive neck nodes treated with irradiation only. Radiother Oncol 22:167-173

9. Curtin HD, Ishwaran H, Mancuso AA, Dalley RW, Caudry DJ, McNeil BJ (1998) Comparison of CT and MR imaging in staging of neck metastases. Radiology 207:123-130

10. King AD, Tse GM, Ahuja AT et al (2004) Necrosis in metastatic neck nodes: diagnostic accuracy of CT, MR imaging, and US. Radiology 230:720-726

11. de Bondt RB, Nelemans PJ, Hofman PA et al (2007) Detection of lymph node metastases in head and neck cancer: a meta-analysis comparing US, USgFNAC, CT and MR imaging. Eur J Radiol 64:266-272

12. Anzai Y, Piccoli CW, Outwater EK et al (2003) Evaluation of neck and body metastases to nodes with ferumoxtran 10-enhanced MR imaging: phase III safety and efficacy study. Radiology 228:777-788

13. Sigal R, Vogl T, Casselman J et al (2002) Lymph node metastases from head and neck squamous cell carcinoma: MR imaging with ultrasmall superparamagnetic iron oxide particles (Sinerem MR) results of a phase-III multicenter clinical trial. Eur Radiol 12: $1104-1113$

14. Baghi M, Mack MG, Hambek M et al (2005) The efficacy of MRI with ultrasmall superparamagnetic iron oxide particles (USPIO) in head and neck cancers. Anticancer Res 25:3665-3670

15. Baghi M, Mack MG, Wagenblast J et al (2007) Iron oxide particle-enhanced magnetic resonance imaging for detection of benign lymph nodes in the head and neck: how reliable are the results? Anticancer Res 27:3571-3575

16. Mack MG, Balzer JO, Straub R, Eichler K, Vogl TJ (2002) Superparamagnetic iron oxide-enhanced MR imaging of head and neck lymph nodes. Radiology 222:239-244

17. van den Brekel MW, Castelijns JA, Snow GB (1998) The size of lymph nodes in the neck on sonograms as a radiologic criterion for metastasis: how reliable is it? AJNR Am J Neuroradiol 19:695-700

18. van den Brekel MW, Castelijns JA, Stel HV et al (1991) Occult metastatic neck disease: detection with US and US-guided fineneedle aspiration cytology. Radiology 180:457-461

19. Wang J, Takashima S, Takayama F et al (2001) Head and neck lesions: characterization with diffusion-weighted echo-planar MR imaging. Radiology 220:621-630

20. Koc O, Paksoy Y, Erayman I, Kivrak AS, Arbag H (2007) Role of diffusion weighted MR in the discrimination diagnosis of the cystic and/or necrotic head and neck lesions. Eur J Radiol 62:205213

21. Kato H, Kanematsu M, Tanaka O et al (2008) Head and neck squamous cell carcinoma: usefulness of diffusion-weighted MR imaging in the prediction of a neoadjuvant therapeutic effect. Eur Radiol (in press)

22. Kito S, Morimoto Y, Tanaka T et al (2006) Utility of diffusionweighted images using fast asymmetric spin-echo sequences for detection of abscess formation in the head and neck region. Oral Surg Oral Med Oral Pathol Oral Radiol Endod 101:231-238

23. Vandecaveye V, De Keyzer F, Nuyts S et al (2007) Detection of head and neck squamous cell carcinoma with diffusion weighted 
MRI after (chemo)radiotherapy: correlation between radiologic and histopathologic findings. Int J Radiat Oncol Biol Phys 67: 960-971

24. Vandecaveye V, de Keyzer F, Vander Poorten V et al (2006) Evaluation of the larynx for tumour recurrence by diffusionweighted MRI after radiotherapy: initial experience in four cases. Br J Radiol 79:681-687

25. Abdel Razek AA, Kandeel AY, Soliman N et al (2007) Role of diffusion-weighted echo-planar MR imaging in differentiation of residual or recurrent head and neck tumors and posttreatment changes. AJNR Am J Neuroradiol 28:1146-1152

26. Takahara T, Imai Y, Yamashita T, Yasuda S, Nasu S, Van Cauteren M (2004) Diffusion weighted whole body imaging with background body signal suppression (DWIBS): technical improvement using free breathing, STIR and high resolution 3D display. Radiat Med 22:275-282

27. Sumi M, Sakihama N, Sumi T et al (2003) Discrimination of metastatic cervical lymph nodes with diffusion-weighted MR imaging in patients with head and neck cancer. AJNR Am J Neuroradiol 24:1627-1634
28. Sumi M, Van Cauteren M, Nakamura T (2006) MR microimaging of benign and malignant nodes in the neck. AJR Am J Roentgenol 186:749-757

29. Abdel Razek AA, Soliman NY, Elkhamary S, Alsharaway MK, Tawfik A (2006) Role of diffusion-weighted MR imaging in cervical lymphadenopathy. Eur Radiol 16:1468-1477

30. King AD, Ahuja AT, Yeung DK et al (2007) Malignant cervical lymphadenopathy: diagnostic accuracy of diffusion-weighted MR imaging. Radiology 245:806-813

31. Fleming I, Cooper J, Henson D (1997) AJCC staging manual, 5th edn. Lippincott-Raven, Philadelphia

32. Hanley JA, McNeil BJ (1983) A method of comparing the areas under receiver operating characteristic curves derived from the same cases. Radiology 148:839-843

33. de Bondt RB, Nelemans PJ, Bakers F, Casselman JW, PeutzKootstra C, Kremer B, Hofman PA, Beets-Tan RG (2008) Morphologic MRI criteria improve the detection of lymph node metastases in head and neck squamous cell carcinoma: multivariate logistic regression analysis of MRI features of cervical lymph nodes. Eur Radiol doi:10.1007/s00330-008-1187-3 